\title{
An unbolted skeleton for primitive surveillance and clientele credentials for impregnable scrutiny chunk
}

\author{
Pro. Marion Kells \\ Department of CSE NTU Singapore
}

\begin{abstract}
-
Data handling and Surveillance in cloud goes through a dynamic and complex hierarchical service chain. Impregnable work is a service that helps to maintain and store the log records additional firmly in the server operative in cloud based atmosphere. Log may be a record of events occurring at intervals associate degree organization's system or network. Log file usually contains additional sensitive and guidance thus it ought to be maintained additional firmly. during this project we tend to discuss the protection problems concerned in log management for a Impregnable work as a service and present style and implementation of a prototype delegating impregnable log manager. Here we tend to impregnable the log records by encrypting it in a very JAR file. The JAR file here can embody a bunch of access management rule specifying whether or not or not and also the means cloud servers and presumably completely different data stakeholder's sq. Measures approved to access the contents itself. Once the authentication succeeds, the service suppliers are allowed to access the data gift at intervals the JAR. Reckoning supported configuration settings that's set throughout the time of creation, the JAR can provide usage management connected to the work, or will provide the alone work utility. As for the work, anytime there is correlate access to the data, the JAR will mechanically generate log records. In this paper, to offer the knowledge surveillance in the cloud and to address integrity and confidentiality problems whereas storing, maintaining, and querying log records JAR file coding is employed.
\end{abstract}

Keywords: JAR creation, log Record generation, AES, Push and Pull Mode.

\section{INTRODUCTION}

Organizations use the Cloud throughout a kind of utterly completely different service models and preparation models (Private, Clientele, and Hybrid). There sq. live selection of 
surveillance issues/concerns associated with cloud computing but these issues represent 2 broad categories: Surveillance problems faced by cloud suppliers and surveillance problems faced by their client. In most of the cases, the suppliers ought to build positive that their infrastructure is impregnable in that their shoppers' data and applications area unit protected whereas the client ought to build positive that their suppliers has taken the right and best surveillance measures to safeguard their knowledge. The intensive use of the virtualization in implementing cloud infrastructure brings distinctive surveillance thought for purchasers or tenants clientele cloud services. Log is a record of events occurring at intervals Associate in Nursing organization's system or network. Log file sometimes contains a lot of sensitive and confidential info therefore it ought to be maintained a lot of firmly. In real world applications sensitive info area unit unbroken in log files in Associate in Nursing untreated machine. The event that once the assailant captures this system we have a tendency to would guarantee that the attacker will gain a touch or no info from the log file and to certain his ability to hack the log file.JAR file may be a compressed file format, wherever you'll be able to store several files. JAR (Java Archive) file format is employed to distribute a sets of java categories. JAR file helps to scale back file size and will collect several files in one by compression it. You will build these jar files viable by aggregation several category file of java applications in it. A jar file will execute from java (Java net Start).JAR file additionally contain some straightforward access management rules that can offer authorization for the users to access the content, A JAR file will be classified into two: Inner JAR and Outer JAR. Inner JAR file contains the encrypted knowledge, category files to facilitate the retrieval of log files and can show boxed knowledge in an exceedingly appropriate format, and a log file for each encrypted item. Outer JAR file can demonstrate user and guide them to the specific inner JAR. As Log files contains a lot of impregnable info, it will be impregnable by encrypting it mistreatment AES rule and storing it within a jar file, Advanced coding customary rule may be a even block cipher wont to shield classified info by Encrypting and Decrypting it. AES rule uses a key (cipher key) whose length is 128, 192 or 256 bits. The cipher secret is distended to into ten, 12, or fourteen spherical keys, severally, exploitation the "Key Expansion" algorithm, wherever every spherical secret is of 128 bits. As AES uses larger key sizes that makes it safer.

\section{LITERATURE SURVEY}

In this section, we have a tendency to have a tendency to first review connected works addressing the privacy and surveillance issues inside the cloud. Then, we have a tendency to 


\section{The American Journal of Applied}

\section{sciences}

VOLUME01 ISSUE01

in short discuss works that adopt similar techniques as our approach but serves for varied functions.

A. Forward Integrity For Impregnable Scrutiny Login this paper, they outline the forward integrity surveillance properties, inspire its appropriateness as system surveillance demand, and demonstrate style that will win this property. Application includes impregnable scrutiny chunk for intrusion detection or answerableness, communication security, and for authenticating partial result of calculation for mobile agents. They discuss regarding impregnable scrutiny log applications in detail and conjointly prove surveillance theorems on forward integrity messages authentication schemes.

B. Guide to laptop Surveillance Log Management Here they give a sensible, real-world steerage for developing, implementing, and maintaining effective log management. This clienteleation covers many topics like the way to establish log management infrastructures, associate in Nursing developing and maintaining strong log management method throughout an organization. The clienteleation conjointly gift log management technology from a highlevel viewpoint, and also the method concerned in log management technologies.

C. Impregnable work As a Service-Delegating Log Management to the Clouding this paper, they establish the challenges gift in an exceedingly impregnable cloud primarily based log management services and propose a skeleton for it.For correct functioning of Associate in Nursing organization its Log Records ought to be firmly maintained for a long amount. Integrity of the log files and the work method ought to be ensured at all the time. In addition, the log files sometimes contain sensitive data, therefore confidentiality and the privacy of log records are vital. However, for deploying a impregnable work infrastructure we have a tendency to want substantial capital expenses therefore several organizations might realize it overwhelming. Empowerment log management to the cloud can seem to be a attainable value saving live.

D. Reliable Delivery and Filtering for Syslog .This paper explains regarding the options that allow the device to be made-to-order for receiving of messages in Syslog. victimization Blocks protractible Exchange Protocol this feature provides impregnable and reliable delivery for syslog messages. Also, it enable multiple session for a single work host freelance of underlying transport strategies, and give the filtering mechanism known as message someone. This module conjointly describes the operate of the Reliable Delivery and Filtering of Syslog feature and the way to tack it in an exceedingly network.

\section{EXISTING SYSTEM}


Data handling is outsourced directly by the cloud service supplier to the alternative entities within the cloud and these entities may delegate the task to others, and so on. Secondly, entities square measure allowed affixing and leaving the cloud in an exceedingly flexible manner. As a result, the knowledge handling within the cloud goes through a posh and dynamic class-conscious service chain that doesn't exist in typical environments. Ordinary internet skeleton. Uses internet services for request and responses. Traditional cryptographic primitives for the purpose of the data surveillance cannot be directly adopted due to user's loss of control of data in Cloud Computing. Therefore, the credentials of proper data storage in the cloud should be done without explicit knowledge of whole data. As each user stores various kinds of data in the cloud and demand for the long term continuous assurance for their data safety, and also the user may often modify the data by deleting or updating it, so the problem for verifying correctness of the data stored in the cloud becomes more challenging.

\section{Limitations}

1) No surveillance for user's knowledge. No authentication or surveillance provided

2) High resource prices required for the implementation.

3) Not appropriate for tiny and medium level storage users.

\section{PROPOSED SYSTEM}

$\mathrm{n}$ this paper, we've got a bent to propose a comprehensive account storing And maintaining log records in AN exceedingly server operational in an cloud-based surroundings. We have a tendency to have a tendency to address surveillance and integrity issues scarcely throughout the log generation half, but together throughout different stages at intervals the log management technique, as well As log assortment, transmission, storage, and retrieval. The key contributions of this paper are as follows. we've got a bent to propose style for the varied elements of the system and develop scientific discipline protocols to handle integrity and confidentiality issues, saving, and question log records at properly, but curious cloud provider and in transit. It provides a shut surveillance analysis and discusses the irresponsibleness and strength of our style at intervals the face of varied nontrivial attacks by implementing Java Running atmosphere. Timer mechanism for limiting the accessing time for surveillance purpose Impregnable JVM for making package tamper resistance capabilities to JAR file. It provides integrity, confidentiality to JAR. AES algorithmic rule give surveillance to the info by 
encrypting it. As AES algorithmic rule uses lager key sizes that makes it safer. Sometimes the Log file contains additional confidential information therefore it ought to be keep firmly. Therefore to supply additional surveillance to the Log file, we have a tendency to cipher it victimization the AES algorithmic rule and store it in an exceedingly jar file.

\section{AES Algorithm}

Advanced cryptography normal is a cruciform key block cipher algorithmic program with a block length of 128 bits. In AES algorithmic program same secret is used for each cryptography and decoding. In AES we are able to use three totally different key lengths: 128 bits, 192 bits, or 256 bits. Cryptography uses ten rounds of process for 128 bit keys, twelve rounds of process for 192 bit keys and fourteen rounds of process for 256 bit keys. All spherical area unit identical except the last round in every case. Every rounds of process involves one single-byte primarily based substitution step, a row-wise permutation step, a column-wise mix step, and also the addition of the spherical key. The order within which these four steps area unit dead is totally different for cryptography and decoding.

\section{AES cryptography and Decryption}

During cryptography, four steps area unit concerned throughout every spherical. Four steps concerned are: 1) Substitute bytes, 2) Shift rows, 3) combine columns, 4) Add spherical key. The last step consists of coring the output of the previous 3 steps with four words from the key schedule.

The four steps concerned throughout the decoding are: 1) Inverse shift rows, 2) Inverse substitute bytes, 3) Add spherical key, and 4) Inverse combine columns. The third step consists of Coring the output of the previous 2 steps with four words from the key schedule. throughout last spherical for cryptography "Mix columns" step is not concerned and throughout last spherical for decoding "Inverse combine columns" step isn't concerned.

\section{CONCLUSION}

Cloud computing permits terribly climbable services to be simply consumed over World Wide net on associate as-needed basis. varied challenges that arise throughout log storage and maintenance in the cloud area unit self-addressed during this paper by proposing a comprehensive resolution. Log files principally contains additional confidential and sensitive information therefore it is maintained firmly by addressing integrity and surveillance issues not solely throughout the log generation phase but additionally throughout the opposite 


\section{The American Journal of Applied}

\section{sciences}

\section{VOLUME01 ISSUE01}

phases within the log management method, together with log assortment, transmission, storage, and retrieval.. In this paper we tend to propose an impregnable resolution by encrypting the log records and storing it within the JAR File. We tend to additionally increase the authentication surveillance by Introducing OTP (One Time Password) throughout every user Operation.

\section{REFERENCES}

[1]Mimi Bellaire_Benet S. Yes, Forward Integrity for Impregnable Scrutiny Chunk.

[2]Karen Kent Scarf one and Mariyah P. Souppaya (2006). "Guide to pc Surveillance Log Management," government agency special Clienteleation.

[3]D. New and M. Rose, " Reliable Delivery and Filtering for Syslog," Request for Comment RFC 3195, net Engineering Task Force, Network unit.

[4]D. Bone and M.K. Franklin, "Identity-Based coding from the Weil Pairing," Proc. Int'I cryptanalytic Conf. Advances in cryptanalytic.

[5]R. Coring, S. Estelle, J.I. den Hertzog, G. Lensing, and I. Stacie, "A Logic for Scrutiny in answerableness in suburbanized Systems," Proc. IFIP TC1 WG1.7 Workshop Formal Aspects in Surveillance and Trust. 